\title{
Sizing for a Special Group of People: Best Practice of Human Body Scanning
}

\author{
Eva Lapkovska \\ Riga Technical University, Faculty of \\ Material Science and Applied Chemistry. \\ Institute of Design Technologies \\ Riga, Latvia \\ eva.lapkovska@rtu.lv
}

\author{
Inga Dabolina \\ Riga Technical University, Faculty of \\ Material Science and Applied Chemistry, \\ Institute of Design Technologies \\ Riga, Latvia \\ inga.dabolina@rtu.lv
}

\begin{abstract}
Due to new circumstances of living, climate and environmental changes, varieties of human body shapes are growing. Therefore, obtaining uniformly clothes for special issues in the group of people with similar interests (dancing groups, choirs, etc.) are getting more and more complex. Besides the self-estimation and perception about the shape and size of the person varies due to different sizing from brand to brand. To dress-up the group of people with different sizes in uniformly way is not an easy task for the supplier - even if the model chosen for the gown is casual, most of the producers doesn't apply a large scale of sizes. Frequently sizing systems do not fit to the needs of the end-users. Size marked on the clothing describes only some information about body size, if any. Therefore, part of clothing supplied is not suitable for end-user groups, but if already purchased it is decided to discard them. Such a set of circumstances, in contrast to global progress towards sustainable development, which is also based on environmental responsibility, can serve as a contributing factor to further growth in clothing consumption. The main purpose of this study is to make an insight into sizing approaches for a special group of people focusing on the best practice of human body 3D scanning. The paper outlines a certain target group's understanding of the clothing size correspondence to their individual body characteristics. Advantages of human body scanning for analysing of body characteristics and solving sizing issues are discussed. Within the study, anthropometric data sets of 50 women group were obtained using a 3D scanner to develop the distribution of this special group into size groups and analyse individual body measurements that are significant for the design of appropriate garment patterns. Conclusions made in this paper acknowledge 3D scanning as an advantageous method for anthropometric data obtaining which are determinate for garment design and sizing system development.
\end{abstract}

Keywords-made to measure, non-contact measurements, sustainable garment producing.

\section{INTRODUCTION}

Recent industry studies within reports explain that today's almost completely linear system for producing, distributing and using clothing is extremely wasteful and polluting. Garment production has approximately doubled over the past 15 years and has been driven by factors such as "fast fashion" phenomenon, rapid change of collections and lower prices. Large amounts of non-renewable resources are consumed by the industry to create clothes that often are worn for a short time period if ever worn [1]. Such trends are not in line with global progress towards sustainable development when development in different areas must meet current needs, but without compromising future generation ability to meet their needs as well [2]. In the garment industry, one of the sustainable development bases - environmental responsibility - cannot be assured by not thinking about the reasons for the steady rise in irresponsible clothing consumption, and certainly about recycling and utilisation issues.

One of the vital factors in the clothing industry is the correct and broad understanding of the end-user anthropometric profile to review and ensure successful clothing production, distribution and use. However, given the constantly changing human body characteristics due to climate and environmental changes, as well as changes in living conditions and quality (obesity problems, medicine and sports development, lifestyle trends, etc.), defining standard sizes that would provide maximum end-user satisfaction is rather difficult [3]. The lack of knowledge about end-user size and shape is the reason for the creation of products that may not be suitable and can be disposed after the first use. Therefore, from the viewpoint of both manufacturers and consumers, reliable and distinctly sizing standards for efficient production circulation are essential [3].

\section{A. Apparel Sizing and Fit}

In mass production, garment patterns are usually adapted from a basic block pattern created to fit a standard average-sized person or a person within the target market. Then patterns are produced in a range of different sizes according to a set of grade rules which are derived from a size chart. The size chart, comprised of body key measurements, is used to define a range of garment sizes within a fashion line It is crucial to ensure fit consistency within the sizing [4]. Manufacturers in different countries use different population data, as latest measurements are too expensive and inaccessible. These tables may be compiled as long ago as they no longer correspond to the real situation, for example, Russian GOST (ГОСТ), and German DOB. Types and number of size designation

Print ISSN 1691-5402

Online ISSN 2256-070X

http://dx.doi.org/10.17770/etr2019vol1.4137 (C) 2019 Eva Lapkovska, Inga Dabolina.

Published by Rezekne Academy of Technologies.

This is an open access article under the Creative Commons Attribution 4.0 International License. 
dimensions and intervals are also derived from the principles and experience of company designers. Sizing principles may be influenced by various tables used over time.

Fit problems caused by sizing of ready-made garments occur within both gender groups and different age groups, also not meeting the needs of individuals with differences in body proportions such as long arms and legs, stooped, normal, and curved stature, large neck circumference, broad shoulders, and people with increased body weight and adipose tissue folds in different areas of the body.

In the industry standards [5], [6], [7], [8], [9], [10] is explained that different systems for size designations and garment labelling are currently used in Europe, highlighting the purpose and need for development. The aim of standardization is to harmonize and simplify the size designation for both consumer benefits and industry in general [5]. Objective is to establish a size designation system that can be used by manufacturers and retailers to indicate to the final consumer (in a simple, direct and meaningful way) body measures ensuring fit of chosen clothing [7]. The third part [5] of the standard is a reference material that includes values and intervals serving as an example of best practice for size designations. In addition, they are developed by the newest sizing surveys in Belgium, France, Germany, Netherlands, Romania, Span and Sweden.

Developers of standards indicate a number of key aspects, for example: the processing of body measurement data as described in this document results in the grouping of body sizes appropriate to the studied population concerned; distribution of body dimensions can change due to changes over times; in order to get a garment-fitting correctly on a body, it is essential to collect the body measurements [8], [9], [10].

Flaws in mass production sizing systems may appear in cases when a specific, sufficiently large group of people need to wear the same style clothing, giving a good overall look in terms of consistent proportions and appropriate fit. Especially if the group has a large variety of body characteristics, it is practically impossible to find clothing for all the group from the mass production, while individual sewing is a time-consuming and expensive process. In such cases, it is necessary to find ways to customize the products according to end-user needs and anthropometric profile, the most appropriate solution is to purchase of made-to-measure clothing.

Made-to-measure clothing is designed specially to fit a person, based on standard sizes and design and adjusted to customer's measurements [11], [12]. In the case of a madeto-measure approach, reliable and sufficiently extensive information on the body measures of wearers is required. The traditional manual methods or contact methods for obtaining anthropometric data are time-consuming and require good skills. Therefore, modern methods of fast and reliable data acquisition - human body 3D scanning, may be the most appropriate solution.

\section{B. Human Body Scanning in Apparrel Industry}

Human body 3D scanning is a modern measuring method that, thanks to its fast and reliable operation, contribute to the development of anthropometric studies and, consequently, to the knowledge about the characteristics of consumer body [13], [14], [15].

Body scanning is a non-contact method when, unlike traditional manual methods, there is no need to touch the human body. Contact methods involve touching a measurer (one or more) with a human body that may confuse the person being measured. As well as considering the duration of the procedure, a person can get tired and change postures, thus creating inconsistent data. Existing research results on the reliability of different types of 3D scanning equipment and data acquisition systems and the usage of gained anthropometric data [15] for pattern design, as well as on permissible measurement deviations mostly show good results [16].

The further use of anthropometric data obtained in 3D scanning is wide in various industries for solving various tasks, and data is mostly easily exportable for different purposes - further quantitative analysis of data, use in CAD systems, work with the virtual human shape/clone (scanatars) in other 3D systems, etc. The repeatability of measurements provided by the scanatar stored in the database is an advantage when it comes to obtaining additional data, for example, if a new/specific product is to be designed or modified design methodology requires the use of additional body measures.

Additional features that are practically impossible with manual measurement methods are exploring the specifics of the body stature, the asymmetry of the body, and the location of the adipose tissue. It is possible with additional measurement tools available in the system, the exact body shape reproduction and the ability to gain cross sections, as well as to superimpose scans. In addition, if the set of measures to be automatically obtained in the system is not complete, there is a possibility to program and later obtain additional measures automatically (for example, in AnthroScan system).

In the apparel industry body scanning is used for: anthropometric sizing surveys, mass customization, fit and shape evaluation, virtual modelling and online fit analysis purposes [17].

For issuing garments to special groups of people with the support of $3 \mathrm{D}$ body scanning and garment $\mathrm{CAD}$ is beneficial in different ways: saves time; reduces costs; gives size recommendations; for sizes out of sizing table scope made-to-measure patterns can be provided; 3D scanatar allows to analyse human body if the shape differs from standardized one; all garments issued are uniformly made and meets end-user needs. Size recommendation and pattern CAD brings new opportunities for inventory and new garment development optimization, virtual prototyping (virtual garment simulation), and online shopping to work on improvements on garment fit and consumer satisfaction [17]. 
Virtual prototyping, compared to traditional real sample sewing, provides opportunities to reduce product development costs (materials, production) and time (production). Critical factors for reliable virtual prototyping are access to appropriate virtual human body shapes and capabilities to define material properties for realistic fabric simulation. Regarding the virtual human body in virtual garment systems also have been developed standards [18], [19]. Studies of different types and approaches confirm the benefits of 3D scanning by obtaining human body reproductions to study and design both casual and special clothing with virtual prototyping techniques. For example, in research about special protective clothing for sport aircraft, a seated 3D body model was used for research [20]. Although still complicated and incomplete, similarly are analysed and evaluated possibilities to design clothing for people with different body shapes and different physical limitations [21].

\section{MATERIALS AND METHODS}

The special group of people in this study includes 50 women who are members of a choir and therefore encounter with a necessity to wear clothing of a united style. Supply of an equally well fit and appearance clothing for members of this collective can obviously be a difficult task given that the participants are aged 19 to 57 years (standard deviation (hereafter - SD) 8,4 years) and differ in terms of body shape: body height 154-181 cm (SD 6,2 $\mathrm{cm}$ ), weight 52 to $132 \mathrm{~kg}$ (SD 19,8 kg), bust circumference 89-139 cm (SD 13,3 cm), waist circumference 66-125 cm (SD 15,3 cm) and hip circumference 91-145 cm (SD 12,5 $\mathrm{cm})$.

Anthroscanner VITUS SMART XXL®) (Human Solutions Group $\mathrm{GmbH}$ ) with AnthroScan data processing system was used to obtain anthropometric data. Hardware and software meet the requirements formulated in ISO 20685 [22]. For every woman, 153 body measurements were automatically retrieved, data processing and compilation were performed for further analysis.

Within the study, the members of the research group were asked to indicate the size of clothing worn in daily life, both upper body and lower body garments. It was done with the purpose of finding out whether the end users themselves have the knowledge and clear approach of finding the appropriate size clothing.

The gained anthropometric data after processing and analysis allowed choir members to be grouped in the appropriate size groups and to compare the distribution with indicated sizes to determine compliance. For size grouping recommendations of EN 13402-3 for size designations and options for range and interval divisions were used [7]. Analysis of individual body measurements within a one-size group of wearers has been performed, selecting body measurements that can play a crucial role in pattern construction of upper body garments for providing fit, traditionally used bust circumference, across back width - armpit level, neck to across back width - armpit level and shoulder width.

\section{RESULTS AND DISCUSSION}

Summarizing the size of everyday-wear items indicated in the questionnaires, it has been observed that wearers are guided by different types of size designations, and, given the differences between manufacturers, some of the respondents have included several designation types. The following types of size designations and their frequency have been discovered, both of upper and lower garments:

a) numeric size - differ from 36, 40, 42, etc. to 52 (upper garments $31 \%$, lower garments $53 \%$ );

b) letter size - differ from S, M, L to XXL (upper garments $5 \%$, lower garments $2 \%$ );

c) different combinations showing that wearers are not confident about the appropriate size or choose different sizes depending on the manufacturer:

- numeric size - two sizes e.g. 38/40 (upper garments $13 \%$, lower garments $20 \%$ );

- $\quad$ letter and numeric size - e.g. M, 40 (upper garments $20 \%$, lower garments $11 \%$ );

- combinations - letter and numeric, e.g. M/L, 38/40 (upper garments $26 \%$, lower garments $9 \%$ );

- completely different size type - e.g. UK size (both type garments $5 \%$ ).

Most popular type used is a number size, nevertheless a relatively large part of group members have indicated combinations (multiple numeric, letter designations), showing an experience of respondents in size meaning diversity. Within this group of people, it serves as proof of confusing differences in sizing systems, which may affect the ability to choose the appropriate size clothing.

For further analysis, a conditional situation when the whole group should choose to clothe from one manufacturer guided by their own perception of the appropriate size is simulated. For this task, each of the indicated size designations is given a consistent explanation by a range in centimetres based on EN $13402-$ 3 size ranges for numeric and letter designations. Already using obtained anthropometric data the size for each subject is determined according to the recommendations of EN 13402-3, for upper garments using bust horizontal girth as a primary dimension and for lower garments waist girth. The aim is to make a comparison and analyse how many of the participants would be able to choose appropriate size clothing in this situation, given the lack of knowledge and understanding of size explanations.

Comparison of garment size distributions (Fig. 1) shows the differences in wearer perceptions and distribution by gained anthropometric data according to EN 13402-3 examples. The most distinct differences for upper garments are in the size groups of ranges 84-92-96, $100-104,110-116$ and 122-128 when part of the subjects does not belong to this group, consequently wearing smaller or larger size clothing. It can be concluded that some part of the subjects may be wrong considering 
belonging to size $84-88$ and 134-140, although they should wear larger size garments for appropriate fit. In the lower body garment distribution comparison significant differences appear in the size group 73-77. For the rest sizes - it can be predicted that a significant part can make a mistake and select a smaller size of lower garments.

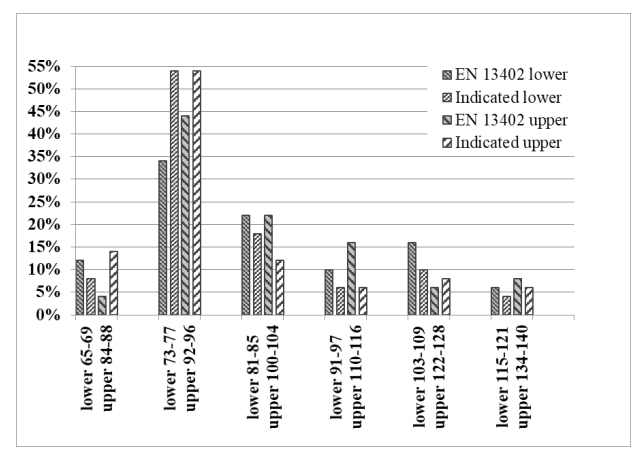

Fig. 1.Comparison of size group distributions of upper and lower body garments by indicated sizes and sizes according to measures.

Experimental comparisons confirm the need to obtain anthropometric data for a full picture of a particular group or population and the ability to develop an appropriate sizing system. Considering the size of the group, the most appropriate method for obtaining anthropometric data is the human body scanning. It is necessary to inform consumers about the size explanations (ranges) so that the end-users knowing main body measurements would be able to choose the most appropriate size.

The analysis described before is based on one primary dimension for upper and lower garments. In a further study, the whole group of subjects was divided into sizes by two measurements (primary and secondary) according to the distributions specified in EN 13402-3. The standard includes an example of division of upper body garment sizes by bust and hip girth ranges. A number of possible proportions of bust and hip girths are shown in the standard, when differnet hip range scales can mach to certain bust range scale ( 88 to 140). The combination of scales depends on the body measurement tables used by the manufacturer or on the availability of anthropometric data of a particular target group.

The distribution of the research participants (Fig. 2) points to the group differences in bust and hip girth proportions. Two scales of hip ranges are rather widely represented, including $21(42 \%)$ and $13(26 \%)$ subjects, however in total resulting in 7 different combinations of the bust and hip range scales. Manufacturers of mass production are not economically interested in producing such a large number of combinations of sizes that require extra time for pattern alterations and stock management without guaranteeing demand for rarely represented sizes. The most represented sizes in the developed table are marked with symbol "*”, for example, 5 participants with a size 96-104 which means compliance with the bust girth interval 94-98 and hip girth interval 102-106.

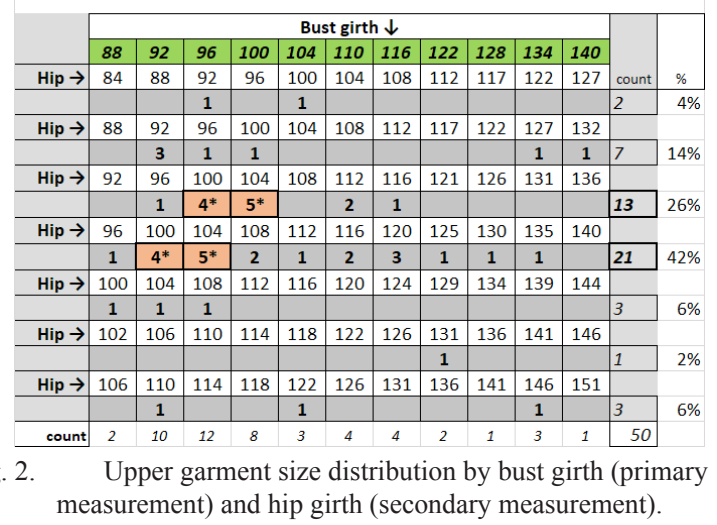

In another example for upper body garments, bust and waist girths are combined. The distribution (Fig. 3 ) reveals two broadly represented scales, including 22 (44\%) and $10(20 \%)$ subjects, but in total illustrates 6 types of proportions within the research participants.

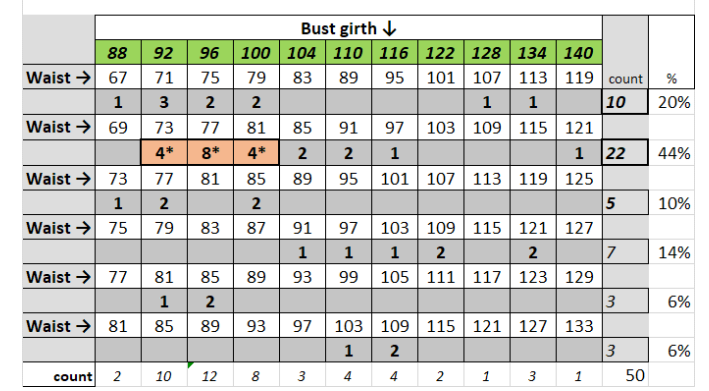

Fig. 3. Upper garment size distribution by bust girth (primary measurement) and waist girth (secondary measurement).

The most represented size within 8 participants is 9677 meaning compliance with the bust girth interval 94-98 and waist girth interval 75-79. Multiple combinations of scales are needed to satisfy the entire group of wearers if the sizes are based on bust and waist girths.

Sizing by hip girth as primary measurement and waist girth as a secondary measurement is an example for lower body garments (Fig. 4). The most represented waist range scale includes 14 (28\%) subjects, and the rest of the total 7 types indicate the differences in hip and waist girth proportions that leads to the production of a large number of different sizes to ensure end-user satisfaction with the fit. The most represented size by 6 participants is 104-77 meaning compliance with the hip girth interval 102-106 and waist interval 75-79.

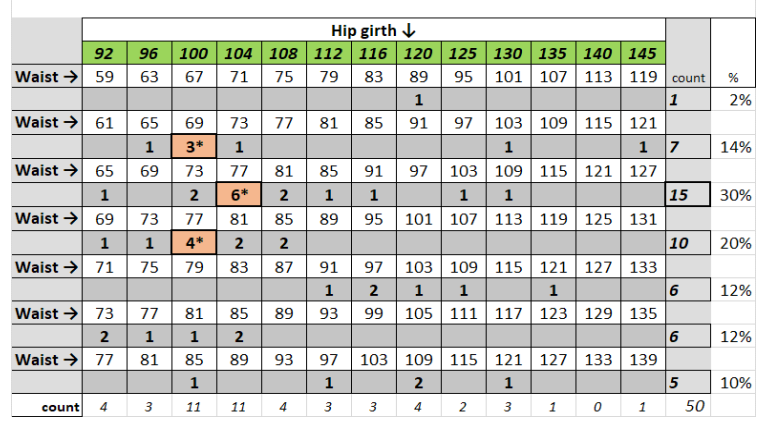

Fig. 4. Lower garment size distribution by hip girth (primary measurement) and waist girth (secondary measurement). 
The production of all the necessary sizes would not be in accordance with the sustainable principles of manufacturing in terms of economic development and resource consumption. The review of the anthropometric profile of end-users is the basis for finding the most adequate solutions for issuing clothing if people are with specific needs and different body characteristics. Therefore, 3D scanning is a prerequisite for fast and reliable anthropometric data acquisition for ensuring the implementation of analysis and usage in sizing and pattern alterations for made-to-measure clothing.

A separate group of people which belong to the most represented size ( 8 subjects) of upper body garments (bust girth range 96 with interval $94-98 \mathrm{~cm}$, waist girth range 77 with interval $75-79 \mathrm{~cm}$ ) were chosen for an insight of value distribution of some individual body measurements relevant for upper garment pattern design. In total 4 basic patterns of close-fitting bodice were developed for the comparison within the study (according to Winifred Aldrich methodology [23]): by standard body measures compliant with EN 13402-3 charts made for women of medium height $(160-172 \mathrm{~cm})$, by the smallest gained values of the 8 subject group, by the largest values of the group and average ones. All the described allowances in the methodology were consistently used for all pattern design.

Body measurement analysis and visual comparison of patterns in Fig. 5 shows that main differences are observed in armscye depth which is defined by the neck to across back width (armpit level) measurement. According to the standard tables used in the methodology armscye depth for bust girth $96 \mathrm{~cm}$ is $21,8 \mathrm{~cm}$, nevertheless, gained measurements show significantly smaller values from 16,5 to $18,3 \mathrm{~cm}$ (average 17,3 cm, SD 0,5 cm). The armhole may be too deep when designing close fitting clothing without additional analyse of the target group measurements.

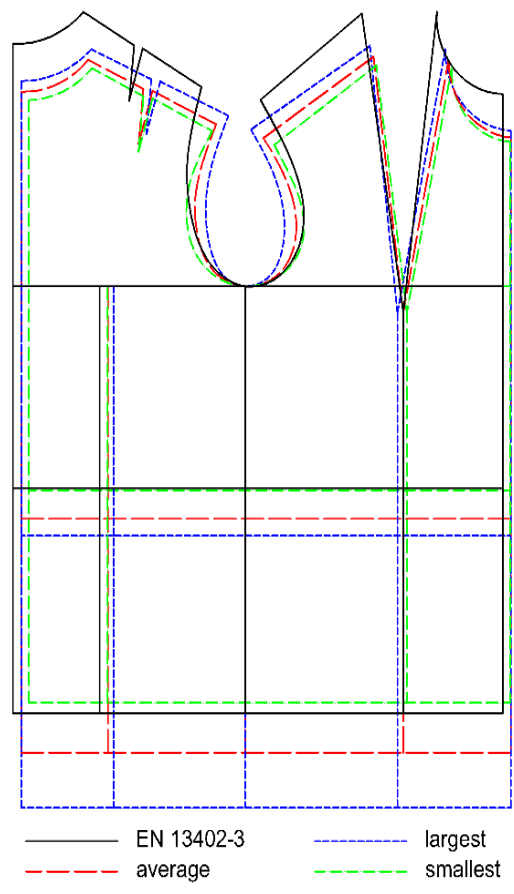

Fig. 5. Basic patterns of close fitting bodice.

The shoulder width, which determines the length of the shoulder, also shows significant differences (Fig. 6). The tables in the methodology compiled according to EN $13402-3$ suggest $12,8 \mathrm{~cm}$ of shoulder length, however body measurement show values from 12,4 to $14,2 \mathrm{~cm}$ (average 13,3 and SD 0,7 cm). Product design by standard tables without additional measurements can result in tightness of the garment in shoulders at least for a part of the group.

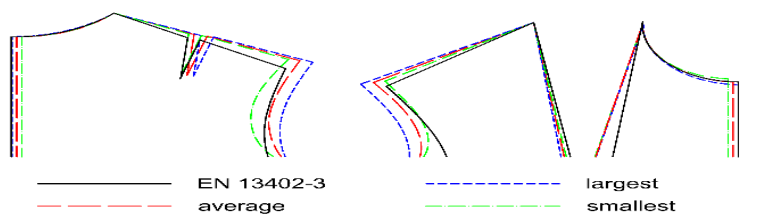

Fig. 6.Copmarison of shoulder length in close fitting bodices.

Differences decisively affecting the pattern structure are observed also in a nape to waist measurements and less important in the waist to buttock measurements. Following the observations, patterns were redesigned by using the most appropriate measurement values to satisfy fit and appearance requirements.

To conclude, the differences in body measurements compared to the standard tables observed in the studied one-size group are significant and may affect the fit and appearance, especially when close-fitting products are provided. Only by gaining a full set of body measurements the analyse is possible for further development of appropriate sizing system and identification of measures for implementation in patterns of made-to-measure clothing.

\section{CONCLUSIONS}

Human body scanning provides a wide range of benefits for the usage in research and improvements of clothing fit. It serves as an effective tool for the rapid and reliable acquisition of anthropometric data of end-users. Without these capabilities, data acquisition for special groups would be difficult when it comes to the need for understanding anthropometric profile and looking for the most appropriate sizing solutions. Initially obtainable information and the possibilities of anthropometric data revision leads to a sustainable approach preventing the production of low-demand sizes and thereby consumption of materials. In addition, the usability of 3D scanning in analyses of body proportions and individual body features as well as in virtual prototyping is growing over time.

The distribution of 50 women by sizes in this research shows the potential difficulties in finding uniform apparel from mass production. The results are evidence to the group's confusion about the differences in sizing systems and their explanations as well as insufficient knowledge about their individual body characteristics for finding the matching size. Differences in close fitting bodice patterns made by standard tables and by real body measurements 
indicate the need for knowledge about anthropometrics of the target group and made-to-measure approaches in garment supply for special groups.

In the cases where a special group of people with different body characteristics needs to be supplied with uniform clothing, human body scanning is the most successful method for anthropometric data acquisition for analysing, for sizing purposes and made-to-measure clothing production.

\section{REFERENCES}

[1] Ellen MacArthur Foundation, A new textiles economy: Redesigning fashion's future, (2017, http://www.ellenmacarthurfoundation. org/publications). [Accessed: Feb. 11, 2019].

[2] European Commission. Sustainable development. Available: http://ec.europa.eu/environment/sustainable-development/index en.htm. [Accessed: Feb. 18, 2019].

[3] R. Nayak and R. Padhye, Eds., Garment Manufacturing Technology. Cambridge, UK: Woodhead Publishing Ltd, 2015, 498 p.

[4] D. Gupta and N. Zakaria, Eds., Anthropometry, apparel sizing and design. Cambridge, UK: Woodhead Publishing Ltd, 2014, 344 p.

[5] Standard. EN 13402-1: 2002 Size designation of clothes - Part 1: Terms, definitions and body measurement procedure.

[6] Standard. EN 13402-2:2002 Size designation of clothes - Part 2: Primary and secondary dimensions.

[7] Standard. EN 13402-3:2017 Size designation of clothes - Part 3: Size labelling based on body measurements and intervals.

[8] Standard. ISO 8559-1:2017 Size designation of clothes - Part 1: Anthropometric definitions for body measurement.

[9] Standard. ISO 8559-2:2017 Size designation of clothes - Part 2: Primary and secondary dimension indicators.

[10] Standard. ISO 8559-3:2018 Size designation of clothes - Part 3: Methodology for the creation of body measurement tables and intervals.

[11] Cambridge Dictionary. Cambridge University Press 2019. Available:https://dictionary.cambridge.org/dictionary/english/made-tomeasure. [Accessed: Feb. 27, 2019].
[12] Merriam-Webster. Merriam-Webster, Incorporated, 2019. Available: https://www.merriam-webster.com/. [Accessed: Feb. 27, 2019].

[13] Standard. ISO 7250-1:2017 Basic human body measurements for technological design -- Part 1: Body measurement definitions and landmarks.

[14] Standard. ISO 20685-1:2018 3-D scanning methodologies for internationally compatible anthropometric databases. Part 1: Evaluation protocol for body dimensions extracted from 3-D body scans.

[15] Standard. ISO 20685-2:2015 Ergonomics - 3-D scanning methodologies for internationally compatible anthropometric databases. Part 2: Evaluation protocol of surface shape and repeatability of relative landmark positions.

[16] C. J. Parker, S. Gill, S. G. Hayes, "3D Body Scanning has Suitable Reliability: An Anthropometric Investigation for Garment Construction", in Proc. of 3DBODY.TECH 2017 - 8th Int. Conf. and Exh. on 3D Body Scanning and Processing Technologies, Montreal QC, Canada, 11-12 Oct. 2017, pp. 298-305.

[17] C. Fairhurst, Ed., Advances in Apparel Production. Cambridge, England: Woodhead Publishing Ltd, 2008, 328 p.

[18] Standard. ISO 18825-1:2016 Clothing - Digital fittings. Part 1: Vocabulary and terminology used for the virtual human body.

[19] Standard. 18825-2:2016 Clothing - Digital fittings - Part 2: Vocabulary and terminology used for attributes of the virtual human body.

[20] A. Rudolf, S. Bogović, B. Rogina-Car, Z. Stjepanovič, S. Jevšnik, A. Cupar, "Virtual prototyping of special protective clothing for sport aircraft pilots", Book of abstracts, 12th Joint International Conference CLOTECH 2017 on Innovative materials \& technologies in made-up textile articles, protective clothing and footwear, October 11th-14th, 2017, Lodz, Poland. Lodz, Poland: Lodz University of Technology, 2017, pp.84-96.

[21] M. Nakić and S. Bogović, "Computational Design of Functional Clothing for Disabled People", Scientifi c Review, Tekstilec 1/2019, 2019, pp. 23-33.

[22] Vitronic. Vitus Bodyscan. Available: https://www.vitronic.com/ fileadmin/user upload/Bodyscannen/Downloads/Englisch/Broschures/VITRŌNIC-VITUSbodyscan-High-Precision-3D-Scans. pdf. [Accessed: Feb. 14, 2019].

[23] W. Aldrich, Metic pattern cutting for women's wear. $5^{\text {th }}$ Edition. Blackwell Publishing, 2008, 218 p. 